\title{
Epistémologies, théories et pratiques professionnelles en communication des organisations
}

Epistemology, Theory, and Professional Practices in Organizational

Communication

Laurent Morillon, Arlette Bouzon et Carolyne Lee

\section{(2) OpenEdition}

Journals

Édition électronique

URL : http://journals.openedition.org/edc/5038

DOI : $10.4000 /$ edc. 5038

ISSN : 2101-0366

Éditeur

Université Lille-3

Édition imprimée

Date de publication : 1 juin 2013

Pagination : $9-26$

ISBN : 978-2-917562-09-3

ISSN : $1270-6841$

\section{Référence électronique}

Laurent Morillon, Arlette Bouzon et Carolyne Lee, « Epistémologies, théories et pratiques professionnelles en communication des organisations », Études de communication [En ligne], 40 | 2013 mis en ligne le 01 juin 2013, consulté le 30 avril 2019. URL : http://journals.openedition.org/edc/5038 ; DOI : 10.4000/edc.5038

Ce document a été généré automatiquement le 30 avril 2019

(c) Tous droits réservés 


\title{
Epistémologies, théories et pratiques professionnelles en communication des organisations
}

\author{
Epistemology, Theory, and Professional Practices in Organizational \\ Communication
}

Laurent Morillon, Arlette Bouzon et Carolyne Lee

1 Depuis plusieurs années, la recherche publique et les mondes socio-économiques sont invités à collaborer, notamment par des exhortations de l'État faites au milieu scientifique d'aller vers l'industrie ${ }^{1}$, et des incitations adressées à cette dernière en vue de contribuer au financement de la recherche publique, via les pôles de compétitivité, le crédit impôt recherche ou des conventions de type $\mathrm{CIFRE}^{2}$, pour ne citer que les plus connus. Mais force est de constater que nous assistons aujourd'hui à une accélération du phénomène, comme le montre la multiplication des occasions de rencontres et d'échanges, parfois de confrontations entre les acteurs concernés, que ce soit à l'initiative du monde académique (au sein de salles de cours, lors de séminaires, colloques ou journées d'études), ou à celle d'acteurs professionnels, d'entreprises, d'associations (tels $\mathrm{P}$ Rocom, Entreprise et communication...). Cette situation va de pair avec un intérêt accru des praticiens pour les travaux de recherche et la faiblesse des dotations financières académiques "de base» imposant aux chercheurs de diversifier leurs sources de financement. Le phénomène est tel que des événements dédiés apparaissent et que certains média s'en font l'écho. Par exemple, un colloque avec pour thème «L'université et le monde professionnel : quels enjeux pour le doctorat? $»^{3}$ est organisé en 2013 et le site Educpros ${ }^{4}$ propose fin 2012 un article sur «Les entreprises en quête de chercheurs interdisciplinaires $»^{5}$. Cette évolution ne va toutefois pas sans heurts et nombreux sont ceux qui en dénoncent les effets pervers ou les déviances induites, telles que la prédominance de la recherche appliquée sur la recherche fondamentale, la priorité donnée au court terme ou au retour sur investissement, etc. Dans un premier temps, nous tentons donc de mieux en comprendre les divers aspects. 
2 En sciences humaines et sociales (SHS), le type de coopération étudié ici est plus récent que dans les sciences dites «dures » dont les frontières entre recherches fondamentale, expérimentale et applicative sont moins établies. Cette collaboration se confronte entre autres à quelques stéréotypes. Certains praticiens estiment en effet que les scientifiques évoluent dans un monde distinct, aux logiques différentes voire opposées aux leurs ${ }^{6}$. Du côté des scientifiques, les principales critiques mettent en avant une relation dite «client-fournisseur » et l'asservissement induit de la science. Pour autant, les exemples de rencontres, constatées lors de la préparation de ce dossier, ne manquent pas. Ainsi en juin 2012 une conférence-débat "Changer de regard-Quand les sciences sociales et les entreprises se rencontrent » a-t-elle été organisée à l'institut des sciences politiques de Paris par l'AFCI et l'APSE ${ }^{7}$, dont l'article issu de cet événement, publié sur le site lemonde.fr, affirme :

Le changement de regard viendra, nous en sommes convaincus, de nouvelles approches. Entre autres, des liens qui nous paraissent fondamentaux entre le monde des praticiens et l'univers des chercheurs. Le régime d'innovation des entreprises a eu très tôt partie liée avec la création de savoirs. Le rôle des sciences, des laboratoires de recherche a été décisif dans les inventions et la réussite des entreprises 8 .

3 Par ailleurs, depuis plus de vingt ans, l'ANVIE ${ }^{9}$ organise des rencontres où scientifiques des SHS et praticiens «qui souhaitent mieux comprendre les évolutions de la société ayant un impact sur l'activité des entreprises $»^{10}$, échangent à partir de résultats de recherches et d'expériences de terrain: "la confrontation de l'expertise des professionnels avec l'analyse des chercheurs est enrichie par les retours d'expérience des participants $»^{11}$. Enfin, les entrelacements entre mondes académiques et professionnels ont, nous semble-t-il, tendance à se «naturaliser» avec des praticiens toujours plus diplômés souvent initiés à la recherche (lors de masters universitaires) ou susceptibles de le devenir par l'entremise de la formation continue.

4 Parmi les SHS justement, les sciences de l'information et de la communication (SIC) occupent un espace scientifique et social riche de zones de contact et d'interfaces avec la société (Boure, 2002). La réflexion sur la praxis y est omniprésente, comme en témoigne par exemple la thématique du $\mathrm{XV}^{\mathrm{e}}$ congrès de la SFSIC ${ }^{12}$, en 2006, intitulée : « Questionner les pratiques d'information et de communication, agir professionnel et agir social ». Dans le champ des SIC, "la demande d'un savoir centré et spécifié aux besoins des professionnels existe et grandit» (Meyer, 2006: 6). C'est le cas spécifiquement en communication des organisations ${ }^{13}$, dont le champ de recherche et les objets d'études induisent des liens étroits avec les praticiens. Or, la communication, qu'elle soit des ou dans les organisations (dite encore "organisationnelle»), est devenue, en France, en l'espace de quinze ans, un objet central d'analyse pour un nombre croissant de chercheurs (Bouzon, 2010), faisant l'objet de nombreux travaux empiriques. La fréquentation des milieux professionnels devient courante que ce soit pour produire des savoirs, pour décrire et éclairer de manière distanciée les pratiques quotidiennes ou pour apprécier des stratégies.

5 La communication est pourtant différemment (pour ne pas dire diversement) considérée dans les milieux académiques et professionnels. Pour certains chercheurs, elle peut être définie tant par les objets - soit les dispositions et dispositifs humains et non humains de communication dans des organisations et/ou constitutives de celles-ci - que par le regard porté sur eux (Bouzon, 2006). Pour les praticiens, elle est avant tout un instrument utile pour résoudre des problèmes, un outil au service de l'organisation qui peut servir à 
faciliter la vente de produits, à renforcer la motivation de salariés ou à développer l'implication de leaders d'influence !

\section{Deux catégories d'acteurs, des projets aux questionnements induits}

Pour autant, ces deux catégories d'acteurs peuvent trouver des intérêts à collaborer.

Du côté des praticiens, les différentes thématiques des travaux scientifiques menés en communication des organisations sont susceptibles de les intéresser (cf. TIC ${ }^{14}$, conduite du changement, gestion de crises, management du personnel, processus de décisions...). Que ce soit pour participer à «fabriquer » les activités ou pour les mettre en perspective, ils mobilisent les théories existantes en vue de produire des connaissances opérationnelles (ou, du moins rapidement opérationalisables) et visent une performativité à plus ou moins court terme. En amont de l'action proprement dite, ils sont attentifs aux recherches susceptibles de leur offrir une perception fine de phénomènes de nature qualitative (représentations, émotions, opinions...), d'introduire de la complexité ${ }^{15}$ dans la description des situations, d'y apporter " un regard différent » voire critique, leur permettant de " prendre du recul ». Cette démarche peut en outre les aider à la formulation de problèmes et d'objectifs, à l'exploration de nouvelles pistes, à l'ouverture de perspectives de pensées et d'actions. Plus en aval de l'action entreprise, le chercheur peut-être invité à intervenir pour garantir l'acte, pour l'évaluer, voire le labelliser (Bouzon et Meyer, 2008).

Quant aux chercheurs, ils souhaitent confronter ces divers savoirs pratiques, notamment les connaissances produites par les praticiens dans l'action, en les mettant en tension avec leurs savoirs académiques afin de les actualiser. Recherches, académique et appliquée, mais aussi interventions expertes de chercheurs peuvent alors s'apporter mutuellement lors de fertilisations croisées (Bouzon et Meyer, 2008). Mais le scientifique se trouve parfois isolé et sans réelle formation dédiée. Il doit alors apprendre à établir des contacts avec des organisations susceptibles de l'accueillir, à négocier les conditions et les contraintes de son engagement, à identifier les méthodologies les plus adaptées, etc. Car en SIC en général et en communication organisationnelle en particulier, le contact avec les organisations (toute unité sociale instituée, marchande ou non) devient indispensable, ne serait-ce que pour les observer et/ou «soutenir des professionnels attachés au développement de leur métier [...] ou de leurs formes organisationnelles » (Meyer, 2006 : 2). Enfin, au-delà de ces considérations, et de manière plus prospective, la valorisation de notre discipline auprès des organisations n'est-elle pas une des conditions pour développer sa reconnaissance au sein de la société?

Les diverses interrogations relatives à la confusion entre postures de recherche et de consultance, amènent à questionner l'utilisation des conclusions des travaux appliqués à des fins scientifiques (Bouzon, 2002), et inversement l'emploi de résultats scientifiques dans des démarches instrumentales. Elles renvoient également à la mise en cause de la tentation économiste susceptible d'entacher l'indépendance du chercheur et/ou la légitimité académique de ses travaux (Heller, 1998), aux questionnements sur les valeurs et modes d'engagements du scientifique (Le Moënne, 1994) mais aussi du praticien au cœur de l'action. Elles ne peuvent aller sans questionnements scientifiques, notamment de la part du chercheur. Ce dernier, par l'entremise d'une indispensable introspection 
intellectuelle, doit en effet être conscient du contexte de sa recherche et de ses valeurs. Il doit identifier les diverses influences subies, fonder ses interprétations, et au final assurer la scientificité de ses travaux par une validité interne et externe.

10 Dans ce contexte, les trois coordinateurs de ce numéro de la revue Études de Communication ont estimé, que, indépendamment des divers discours (parfois passionnés) que suscite un tel sujet, il est une place pour une lecture scientifique des processus communicationnels existants entre les divers acteurs concernés ; mais aussi qu'une étude distanciée des effets croisés de leur collaboration effective, qu'elle soit voulue ou imposée, est opportune. C'est pourquoi l'appel à articles a invité les auteurs à aborder ces questions dans une problématique communicationnelle, ou qui verrait l'objet « communication » occuper une place centrale, en intégrant une obligatoire dimension empirique. Sur la trentaine de contributeurs de disciplines différentes qui ont proposé un résumé initial, seuls six articles ont été retenus, sur la base d'un texte intégral présenté anonymement à l'expertise. Le présent numéro, pluridisciplinaire, rassemble in fine des articles qui font état de recherches conduites dans des directions distinctes, dont les objets, cadres d'analyse ou démarches de recherches sont pluriels... se faisant ainsi le reflet de la complexité des phénomènes abordés.

\section{Questionner les rapports entre pratiques et théories}

11 C'est dans le cadre d'une actualité riche en collaborations de toutes sortes que les coordinateurs (deux chercheurs français et une chercheure australienne) de ce dossier ont donc souhaité questionner les rapports entre pratiques professionnelles et théories, en focalisant leur propos sur la communication des organisations. Cette démarche a semblé d'autant plus pertinente que les évolutions sont notables, de part et d'autre.

Depuis les premières études académiques portant sur la communication des organisations, dans les années 1940 aux États-Unis, les évolutions ont, en effet, été nombreuses. Dès les années 1960, l'étude de ces phénomènes acquiert un statut scientifique affirmé, notamment en économie. A l'origine, la plupart de ces recherches dédiées à l'entreprise servent alors la création de savoirs et l'expérimentation de nouveaux processus de travail pour permettre l'accroissement de la productivité des salariés (Bouzon, 2010). Il faut attendre les années 1990 en France mais aussi en Australie, pour observer l'émergence d'un champ dédié et l'usage de l'expression « communication des organisations » en France et « organizational communication » en Australie. Or peu à peu, les recherches se sont élargies à d'autres organisations: associations, collectivités territoriales, institutions...

13 Progressivement, à côté des modèles prédictifs dominants utilisés par les sciences de gestion, les SIC françaises se distinguent par l'adoption de modèles explicatifs pluridimensionnels, parfois sur fond de posture critique (Morillon, Aldebert et Szafrajzen, 2010). Cette dernière, si elle existe peu ou prou dans d'autres pays, s'exerce quelque peu différemment en Australie où la perspective fonctionnaliste reste prédominante, en raison d'une pression, parfois subtile, mais avérée du marché. Les chercheurs y oscillent ainsi de manière relativement peu explicite entre ces postures critique et fonctionnaliste, réfléchissant quelquefois à des modèles prédictif et/ou de contrôle. Aujourd'hui, les paradigmes épistémologiques positiviste, interprétativiste et constructiviste sont usuellement (et quelque peu schématiquement) identifiés (Thiétart, 2003). La première perspective appréhende la communication selon le modèle télégraphique (Shannon et 
Weaver, 1975) : l'information, susceptible de subir des «bruits », est codée puis transmise via un "canal» d'un émetteur vers un récepteur. Les modèles cybernétiques et systémiques, tout en s'inscrivant dans cette visée, ont complexifié par la suite ce modèle «de base» (Giroux, 1994). Dans la perspective interprétativiste, le projet est compréhensif et l'intérêt porte sur la motivation des individus en présence. Les capacités cognitives, affectives et stratégiques dont ils sont pourvus modifient la nature des interactions et des interprétations individuelles. Le rapport à la connaissance, à l'inverse du positivisme, est ici relativiste. Enfin, dans cette même posture quant à la dépendance du sujet et de l'objet, la perspective constructiviste se focalise sur les finalités et considère plus particulièrement les phénomènes de (co)construction du sens. L'organisation y est notamment considérée comme un produit de la communication (Cooren, Taylor et Van Every, 2006).

14 Aujourd'hui, nombreux sont les chercheurs qui, en France ou à l'étranger, explorent les potentialités des paradigmes interprétativiste et constructiviste. Un tournant discursif a émergé en France et certains chercheurs, dans la foulée de l'École de Montréal, développent des travaux se référant aux théories et méthodes de l'organizational Communication (Putnam et Nicotera, 2009). Carolyne Lee de l'université de Melbourne, cocoordinatrice du présent dossier, se référant aux travaux de Simpson and Zorn (2004) indique qu'en Australie et en Nouvelle-Zélande, la communication est considérée comme constitutive de l'organisation davantage que comme un phénomène se déroulant " dans " les organisations. C'est sans doute pourquoi ces deux termes sont fréquemment " désaccouplés » dans la cinquantaine d'articles académiques australiens et néo-zélandais que les auteurs Simpson et Zorn ont étudié et comparé à ceux produits en Amérique du Nord. Leur étude montre également un intérêt marqué porté par les chercheurs aux objectifs et aux intérêts de certaines parties prenantes marginales de l'organisation.

Les communicants praticiens (responsable et chargé de communication interne et/ou externe, attaché de presse, consultant...) sont, quant à eux, confrontés depuis les années 1970 à la mondialisation, à une concurrence accrue, à une globalisation des secteurs d'activités, à une exigence financière croissante de l'actionnariat ainsi qu'à des crises financière et économique récurrentes. Ils doivent s'adresser à des publics (salariés, consommateurs...) plus diplômés, plus informés, plus cultivés, plus qualifiés et plus critiques (Sureau, 1995). Pour ces praticiens, l'organisation, ses problèmes et surtout ses résultats sont au centre des préoccupations. De fait, ils adoptent majoritairement une approche fonctionnaliste et plus particulièrement le modèle "marketing " (Mucchielli et Guivarch, 1998). Issu des sciences de gestion et conçu pour l'action, celui-ci rationalise et instrumentalise une communication censée résoudre des problèmes: définition d'une situation, détermination d'objectifs à atteindre, identification de cibles, mise en œuvre de moyens, contrôle des discours et évaluation des résultats (Morillon et Gramaccia, 2013). Influencé par certains travaux de la sociologie des médias, ce modèle de mercatique emprunte quelques uns de ses principes au modèle de la «communication à deux niveaux " et à la théorie de l'information (Johansen et Frandsen, 1998). Or, certains praticiens, eux-aussi confrontés à ce dynamisme de l'environnement, à la diversité des acteurs ainsi qu'à la relative efficacité des modèles prédictifs s'ouvrent à d'autres théories, qu'elles soient descriptives ou prescriptives. Ils n'hésitent plus, alors, à se tourner vers les chercheurs en SHS et à interpeller les SIC.

Ce constat, qui nous amène à revisiter les situations vécues, se traduit en un ensemble, non exhaustif, de questions d'ordre épistémologique, théorique, pragmatique ou encore 
méthodologique : quelles sont les fertilisations croisées actuelles et potentielles entre théories et pratiques? Quels sont aujourd'hui les modèles qui structurent les pratiques notamment suite aux évolutions des théories des organisations (Rojot, 2003) ? Au-delà du paradigme fonctionnaliste et du modèle «marketing », quelle est et/ou pourrait-être la place des réflexions pour ceux issus des paradigmes émergents? L'adoption d'une approche constructiviste dans les pratiques est-elle tenable compte tenu des présupposés sur lesquels elle repose (Mucchielli ${ }^{16} \ldots$ ) ? Les chercheurs en sciences sociales peuvent-ils (doivent-ils) résoudre des problèmes d'organisations, participer à une rationalisation et/ ou une instrumentalisation de la communication, voire de la science ? Notre projet n'est pas ici de comparer, pour hiérarchiser ou opposer, le statut et la légitimité des savoirs issus des recherches fondamentales et ceux liées aux recherches appliquées. Il ne s'agit pas non plus de débattre, dans une posture qui se voudrait critique, sur une marchandisation du savoir. Le projet de ce dossier s'avère tout à la fois davantage ambitieux et plus modeste. Il s'agit dans la présente livraison de questionner, voire mettre en tensions, les pratiques et les soubassements théoriques existants en communication des organisations, tant en France qu'à l'international. Pour cela, les six articles, préalablement sélectionnés sur cette base, ont bénéficié d'une démarche constructive de relectures et d'améliorations plus ou moins substantielles. Nous tenons ici à remercier les évaluateurs dont l'implication a permis la constitution de ce dossier qui comporte à la fois des articles en sciences de l'information et de la communication bien sûr mais aussi en sociologie et en sciences de gestion. Les contributions, si elles sont effectivement internationales, demeurent cependant francophones, venant de France, de Belgique et du Canada.

Un des intérêts de ce dossier est de réunir des articles qui font état d'un retour réflexif, distancié, sur certaines recherches menées. Afin de présenter la diversité des démarches et la richesse des réflexions induites, nous avons choisi d'organiser les contributions selon deux axes. Le premier composé de trois textes questionnent les fertilisations croisées entre théories et pratiques tandis que les trois autres, explorent dans un deuxième axe les potentialités des épistémologies constructivistes, susceptibles de renouveler les pratiques des chercheurs et/ou des praticiens.

\section{De la théorie à la pratique : fertilisations croisées}

Dans la première partie de ce dossier, dédiée aux réflexions sur les liens et les apports mutuels entre pratique et théorie, les textes sont présentés selon le degré d'implication $\mathrm{du}$ chercheur vis-à-vis de la pratique professionnelle. Cette question de la distance par rapport à l'objet, et d'une manière générale du rapport intellectuel au monde, est en effet essentielle dans les sciences sociales. Si dans une perspective expérimentaliste, l'objectivité est (a priori) garantie par la non implication du chercheur vis-à-vis de son objet, en sciences sociales, l'extériorité s'avère être en soi une forme d'engagement et induit en partie la nature de la connaissance. Pour Elias (1993), engagement et distanciation sont deux opérations intellectuelles dont la confrontation permet le rapport au monde : l'engagement, caractérisé par le subjectif offre l'ancrage dans le terrain social tandis que la distanciation l'objective par une démarche intellectuelle de recul critique. Les auteurs des trois textes suivants proposent donc, à partir de postures certes différentes, de mettre au jour puis de questionner les apports mutuels de la science et de 
la pratique professionnelle. Ils explicitent certaines des tensions existantes entre enjeux sociaux et enjeux épistémologiques, entre implication et explication.

Ainsi Denis Benoit et Didier Courbet s'intéressent-ils au neuromarketing qui instrumentalise les neurosciences afin d'améliorer les stratégies de communication commerciale des entreprises. Ils se proposent d'observer les études menées par certains praticiens de la publicité pour questionner la «manipulation» commerciale et publicitaire, l'influence réelle du neuromarketing et les tensions éthiques induites. Ils recensent notamment les mythes associés à cette démarche "innovante » usant des techniques issues de la psychologie expérimentale. Si les auteurs démontrent une efficacité finalement toute relative, ils n'en invitent pas moins d'autres chercheurs à participer à ces réflexions sur le libre arbitre et l'identité individuelle, le jugement moral et l'éthique de cette « discipline ». Denis Benoit et Didier Courbet, en ne prenant pas part à la pratique du neuromarketing, adoptent ici une position distanciée. Si celle-ci peut apparaitre relativement "confortable " pour élaborer la connaissance, les chercheurs n'en demeurent pas moins engagés comme sujet connaissant et comme sujet citoyen. Ils ne peuvent donc scotomiser les débats qui animent la société. L'objectivation complète et l'absence de jugement de valeur s'avèrent donc in fine impossibles.

20 Serge Agostinelli et Marielle Metge proposent quant à eux de questionner une solution de continuité entre théorie et pratique. Ils postulent en cela que l'abstraction est au cœur de l'action humaine et qu'elle ne disqualifie pas la théorie face à l'évidence des pratiques. Pour illustrer leur propos, les auteurs présentent une recherche située qui a pour finalité la création d'un réseau de femmes chefs d'entreprises sur la région marseillaise. Si la demande vient du terrain, la recherche est paradoxalement théorique. Cette dernière permet à la fois de transformer la réalité (le terrain) et de produire des connaissances concernant ces transformations (la recherche). Ce cas permet d'engager une discussion sur les conditions de la production de connaissances pour l'organisation ainsi que sur la genèse et la " racontabilité » des connaissances professionnelles. Au final, c'est la manière dont s'opère la fertilisation croisée entre recherche et action qui est ici discutée. Cet article propose donc à la réflexion de se centrer sur un continuum théorie / pratique. Il peut offrir un cadre d'analyse, ou du moins une clef de lecture, pour les autres textes du présent dossier.

21 Marlène Dulaurans et Olivia Foli, qui ont mené leur thèse avec une Convention Industrielle de Formation par la REcherche (CIFRE), exposent certains des ajustements qui se sont révélés nécessaires lors d'immersions de longue durée et leurs effets sur les connaissances produites. L'organisation, entre évolutions structurelles, épreuves pratiques et relationnelles, devient sans aucun doute un contexte contraint, a fortiori pour un jeune chercheur. C'est à partir de leurs deux thèses, l'une menée en SIC, l'autre en sociologie, que les auteurs explicitent comment elles se sont accommodées de ces contraintes et des tensions existantes (employeurs ignorant la recherche, décisions contrariantes, divergences entre exigences académiques et professionnelles, etc.). Afin de maintenir la fertilité, la pertinence et la scientificité de la connaissance produite, les auteurs mettent au jour les adaptations continues, les changements de posture, l'inventivité méthodologique et la curiosité vis-à-vis des phénomènes originaux tout en tenant « le cap » d'un paradigme initialement privilégié. Cet article nous montre en outre que la mise en œuvre de méthodes ne dépend pas que de critères rationnels définis $a$ priori mais qu'elle s'ajuste finalement aux spécificités du terrain et aux attentes des acteurs (Bouzon et Meyer, 2008). 

constructivisme donnant à réfléchir sur ses potentialités, tant d'un point de vue académique que professionnel.

\section{Convention constructiviste et pratique professionnelle}

Dans la pratique professionnelle, si la plupart des consultants appliquent des méthodologies prédéfinies (Petitet, 2004) et apportent «des conclusions et des recommandations sinon conformes à ce que pourrait attendre leur client, du moins cohérentes avec sa façon de poser le problème » (Le Moënne, 1991: 166), tel n'est pas le cas des chercheurs a priori. Ceux-ci bénéficient en effet d'un projet ${ }^{17}$, d'outils conceptuels, d'un statu $\mathrm{t}^{18}$ et de délais différents, qui sont susceptibles de renouveler les approches mises en œuvre. Un nombre croissant de démarches relevant du constructivisme apparaissent aujourd'hui dans des recherches appliquées voire dans des recherchesactions (Morillon, Aldebert et Szafrajzen, 2010). Le constructivisme - ou plutôt la convention constructiviste (Le Moigne, 1990) tant les postures sont dans les faits variées postule que l'organisation est une construction (principe de «l'univers construit»), qui englobe le sujet qui tente de la maîtriser ou se contente de l'observer à travers la représentation qu'il s'en fait (principes de "représentabilité » et de " projectivité »), a un fonctionnement complexe qui ne peut se décomposer en éléments simples indépendants et ne peut conduire qu'à des solutions plus ou moins satisfaisantes (principe de «l'action intelligente ») (Bouzon, 2002). Ainsi, les trois textes présentés dans cette partie (auquel pourrait être ajouté celui de Serge Agostinelli et Marielle Metge), tendraient à prouver la viabilité de l'adoption d'une épistémologie constructiviste dans certaines pratiques professionnelles. Cependant, les potentialités en matière de "fertilisations croisées", notamment en ce qui concerne la production d'actions, sont différentes en fonction du rapport aux praticiens des auteurs-chercheurs ici présent. Elles sont en effet de natures, voire de temporalités différentes. Car si certains n'ont aucun contact direct avec les praticiens, d'autres en observent les pratiques avec un regard distancié et/ou répondent à une commande. Ces trois cas de figure, finalement assez représentatifs des possibilités offertes au chercheur, sont présentés dans la partie suivante.

Dans son article, Franck Cochoy défend l'intérêt de l'étude de la presse professionnelle, notamment pour sa capacité à rendre compte de l'évolution de leur monde, et ce sans discussion préalable de la "véracité » de ce qu'elle rapporte. Pour justifier cette entrée par une source unique, il affirme notamment, dans une posture constructiviste, que les faits et les discours sont produits simultanément. Ainsi, le média considéré, s'il rend compte d'un point de vue particulier, ne peut être réductible à une seule instance. Cette approche, nommée «focalisation médiale » par son parti-pris est susceptible d'enrichir des réflexions d'ordre méthodologique. Pour illustrer son propos, Franck Cochoy rend compte d'une analyse historique centrée sur la revue américaine Progressive Grocer qui, à partir des années 1920, promeut de nouvelles techniques commerciales auprès d'épiciers indépendants. L'auteur, en s'intéressant à l'« objet langagier de communication » (Delcambre, 2000), accède à certaines représentations, repère la construction collective du sens de l'activité et observe indirectement certaines des pratiques dans les milieux professionnels. De fait, ces outils de communication professionnelle, qui peuvent être appréhendés comme des objets scientifiques, constituent une voie d'accès privilégiée à l'analyse des organisations (Heller, 2000).

Études de communication, 40 | 2013 
uelo Vásquez, Benoit Cordelier et Viviane Sergi, se proposent d'étudier quant à eux la production de pratiques et d'artefacts contribuant au développement d'une image de marque. Convoquant la sociologie d'association (entre humains et non humains) de Latour, ils considèrent de manière originale la dimension organisante de la marque. Les auteurs identifient comment une approche constructiviste, plus particulièrement processuelle, permet de renouveler l'étude des pratiques professionnelles dans le domaine de la production de l'image de marque (ou branding). Dépassant les traditionnelles sphères commerciale, économique ou juridique, ils questionnent le travail d'assemblage et de traduction qui aboutit à cette image de marque. Ils illustrent leur propos par l'entremise d'une étude de cas d'un travail de branding porté par le corps professoral d'un département de l'université canadienne dont ils font partie. Considérée comme pratique organisationnelle, cette étude de la marque ouvre de nouvelles perspectives dans la manière d'analyser certaines pratiques mercatiques.

Enfin, François Lambotte et Philippe Scieur mettent au jour puis analysent dans leur article la « cuisine » de l'intervention de chercheurs en organisation. Ils questionnent à la fois le statut de l'intervention, celle de son incidence sur l'organisation et celle de l'intervenant. L'analyse porte plus particulièrement, dans cet article, sur le rapport final de l'audit organisationnel. Les auteurs s'interrogent notamment sur le rôle que peut jouer son écriture, et la négociation sous-jacente, dans le passage d'un audit à une autre forme d'intervention prolongeant le travail réalisé. Ils s'inscrivent ouvertement dans un paradigme épistémologique constructiviste et plus particulièrement dans une sociologie dite de la traduction. La réflexion porte en effet sur la textualisation, soit le processus qui relie conversations et textes. La réflexion est fondée sur plusieurs expériences, dont un cas récent d'intervention en organisation. Cette description du processus d'écriture, notamment de l'interaction entre conversations et textes, se révèle originale et féconde.

\section{Pour conclure}

Les six articles présentés dans la présente livraison de la revue Études de communication témoignent, s'il en était besoin, de la pluralité des projets, des objets, des cadres d'analyse mais aussi des démarches de recherches, notamment des expériences méthodologiques et des théories mobilisées. Certains auteurs de ce dossier proposent des réflexions de nature méthodologique ou épistémologique, d'autres s'intéressent aux pratiques de manière distanciée quand quelques-uns s'investissent dans des recherches appliquées voire dans l'action. Pour ces derniers qui mobilisent le constructivisme, une question importante demeure. Quid en effet du dépassement, lors de l'opérationnalisation de la recherche dans une visée transformatrice de l'organisation, du (néo)fonctionnalisme?

Mais si les disparités entre les situations sont réelles, ce dossier ne manque pas de cohérence. Car l'ensemble de ces chercheurs, en proposant un retour réflexif sur leur propre pratique, font état des liens qu'ils ont observés, créés, induits (qui parfois, d'ailleurs, les ont contraints) entre les théories mobilisées et les pratiques professionnelles observées.

Pour conclure, ces réflexions sur la recherche " en train de se faire » et notamment sur l'ouverture peu courante des «boites à outils » du chercheur, permettent l'émergence de problématiques originales dans les rapports entre savoirs et pratiques. Finalement, la posture réflexive adoptée ici est susceptible, par la description puis l'analyse, de

Études de communication, 40 | 2013 
participer à la compréhension de certaines des nouvelles formes de relations d'échanges et de collaborations entre praticiens et chercheurs. La lecture des présentes contributions peut également éclairer certaines des mises en tensions entre pratiques professionnelle et de recherche.

\section{BIBLIOGRAPHIE}

Almeida (d') N. et Andonova Y., (2006), La communication des organisations, in Olivesi S. (dir.), Sciences de l'information et de la communication. Objets, savoirs, discipline, Grenoble, PUG, pp. 129-143, 286 p.

Boure R., (2002), Les origines des sciences de l'information et de la communication. Regards croisés, Lille, Presses Universitaires de Septentrion, $184 \mathrm{p}$.

Bouzon A., (2002), Communiquer dans l'incertain, la communication dans les processus de conception innovante à « risques maîtrisés », Mémoire d'habilitation à diriger des recherches, LERASS, Université Toulouse III, $339 \mathrm{p}$.

Bouzon A., (2004), La place de la communication dans la conception de systèmes à risques, Paris, L'Harmattan, collection Communication des organisations, $242 \mathrm{p}$.

Bouzon A., (2006), Étudier la communication organisationnelle : champs, concepts, perspectives, Paris, L'Harmattan, collection Communication des organisations, 298 p.

Bouzon A., (2010), Las investigaciones en comunicación de las organizaciones. Orígenes y fundamentos, in Second congrès International de la Asociación Española de Investigadores de la Comunicación (AE-IC), Comunicación y desarrollo en la era digital, Faculté des sciences de la communication de l’Université de Málaga, Espagne, cédérom.

Bouzon A. et Meyer V., (2008), La recherche action en communication organisationnelle, Paris, L'Harmattan, Collection communication et civilisation, $230 \mathrm{p}$.

Cooren F., Taylor J. R. et Van Every E. J., (2006), Communication as organizing : Empirical approaches to research into the dynamic of text and conversation, Mahwah, NJ, Lawrence Erlbaum, $248 \mathrm{p}$.

Elias N., (1993), Engagement et distanciation, Paris, Fayard, 258 p.

Giroux N., (1994), La communication interne : une définition en évolution, in Communication et organisation, $n^{\circ}$ 5, Bordeaux, pp. 17-46.

Gold R., (2003), Jeux de rôles sur le terrain. Observation et participation dans l'enquête sociologique, in Céfaï D. (dir.), L'enquête de terrain, Paris, La Découverte, Recherches - La bibliothèque du M.A.U.S.S., 630 p.

Heller T., (1998), Le chercheur face à la communication d'entreprise, in Le Moënne C. et al., Communications d'entreprises et d'organisations, pp. 13-26, Rennes, Presses Universitaires de Rennes, $235 \mathrm{p}$.

Laramée A. et Vallée B., (1991), La recherche en communication, éléments de méthodologie, Québec, Presse de l'Université du Québec, 378 p. 
Le Moënne C., (1991), Le rôle des consultants en communication, in Les cahiers du Lerass, Toulouse, $n^{\circ} 23$, pp. 157-174.

Le Moënne C., (1994), Les sciences sociales au risque de l'expertise commerciale ?, in Sciences de la société, 32, Toulouse, pp. 5-10.

Le Moigne J. L., (1990), Epistémologies constructivistes de sciences de l'organisation, in Martinet A. C., Epistémologies et sciences de gestion, Paris, Economica, pp. 81-140, 249 p.

Lewin K., (1943), Forces Behind Food Habits and Methods of Change, in Bulletin of National Resources Council, $\mathrm{n}^{\circ} 108$, pp. 35-65.

Meyer V., (2006), De l'utilité des recherches-actions en SIC, in Communication et organisation, 30, en ligne : http://communicationorganisation.revues.org/3455, consultation le 18 janvier 2013.

Morillon L., (2008), De l'idylle au détournement, quels apports des CIFRE en Sciences de l'Information et de la Communication?, in $\mathrm{XVI}^{\mathrm{e}}$ congrès SFSIC, Les sciences de l'information et de la communication : affirmation et pluralité, Compiègne, 11-13 juin, actes en ligne : http:// www.sfsic.org/congres_2008/spip.php?article42.

Morillon L., Aldebert B. et Szafrajzen B., (2010), Pour une lecture croisée des recherches sur la communication des organisations en sciences de l'information et de la communication et en sciences de gestion, in XVII ${ }^{\mathrm{e}}$ congrès SFSIC, Dijon, 23 au 25 juin 2010, actes en ligne : http:// tabarqa.ubourgogne.fr/outils/OconfS/index.php/SIC/SFSIC17/paper/view/88/8.

Morillon L. et Gramaccia G., (2013), Pour une approche critique des modèles dans les manuels de communication des organisations, in Heller T., Huet R. et Vidaillet B. (coords.), Communication, organisation et pensées critiques, Lille, presses universitaires du septentrion, pp. 375-385, 460 p.

Mucchielli A., (2001), La communication interne, les clés d'un renouvellement, Paris, Armand Colin, $206 \mathrm{p}$.

Mucchielli A. et Guivarch J., (1998), Nouvelles méthodes d'étude des communications, Armand Colin, Paris, $174 \mathrm{p}$.

Petitet V., (2004), Communication et domination dans les organisations : analyse d'un cabinet conseil, Thèse de doctorat en sciences de l'information et de la communication, ENS, Lyon.

Putnam L. L. et Nicotera A. M., (2009), Building theories of organization : The constitutive role of communication, New York : Routledge, $240 \mathrm{p}$.

Rojot J., (2003), Théorie des organisations, Paris, Eska, 541 p.

Shannon C. et Weaver W., (1975), Théorie mathématique de la communication, Paris, CEPI, 188 p.

Simpson M. et Zorn T., (2004), Locating the frontiers of organisational communication scholarship in Australia and New Zealand, in Australian Journal of Communication, vol. 31, issue 3, pp. 13-34.

Thiétart R. A., (2003), Méthodes de recherche en management, $2^{\mathrm{e}}$ édition, Paris, Dunod, 537 p.

\section{NOTES}

1. Cf. par exemple le "Rapport Berger» du 17 décembre 2012, destiné au Président de la république qui incite à développer les coopérations entre la recherche publique et la recherche privée (propositions 56 à 58, 102 et 103).

2. Convention Industrielle de Formation par la REcherche : subventionne des organisations qui embauchent un doctorant. 
3. Colloque organisé au CNAM dans le cadre du Labex HASTEX les 24 et 25 janvier 2013.

4. Site de l'Étudiant, destiné aux professionnels de l'enseignement supérieur.

5. http://www.letudiant.fr/educpros/actualite/chercheurs-misez-sur-l-interdisciplinarite.html consultation le 20 septembre 2012.

6. http://www.lemonde.fr/idees/article/2012/07/10/entreprise-il-est-temps-de-changer-deregard_1730880_3232.html ?xtmc=charpentier\&xtcr=1 - consultation le 29 septembre 2012.

7. Association Française de Communication Interne et Association des Professionnels en Sociologie de l'Entreprise.

8. Ibid., http://www.lemonde.fr.

9. Association Nationale de Valorisation Interdisciplinaire de la recherche en sciences humaines et sociales auprès des Entreprises.

10. http://www.anvie.fr/pages/qui-sommes-nous - consultation le 29 septembre 2012.

11. Ibid., http://www.anvie.fr.

12. Société Française des Sciences de l'Information et de la Communication.

13. Le choix de l'expression « communication des organisations » se veut ouvert. Il n'exclut pas les autres considérations de la communication, notamment organisationnelle. Pour un panorama cf. D’Almeida et Andonova, 2006.

14. Technologies de l'Information et de la Communication.

15. «Remettre du savoir, de l'intelligence, du complexe dans un univers qui a été par trop réduit [...] dépasser la réduction opérée par la corporate gouvernance [...] sortir d'un prêt à penser managérial défaillant », ibid., http://www.lemonde.fr.

16. "Ce point de vue présuppose que les acteurs ont la volonté de dialoguer pour rapprocher leurs points de vue. Il présuppose aussi que le management s'efforce de piloter, d'une manière non manipulatoire, les échanges. Il présuppose encore et en outre que les points de vue sont 'rapprochables' (Mucchielli, $2001: 11)$.

17. Si le chercheur peut aider l'organisation, il est avant tout un scientifique. Il se doit en tant que tel de rechercher prioritairement le plus haut degré de vérité dans la description, l'explication ou l'interprétation d'un phénomène (Laramée et Vallée, 1991).

18. Chercheur et consultant ne bénéficient ni de la même image ni de la même place dans les rapports de force existants dans l'organisation (Heller, 1998).

\section{RÉSUMÉS}

La multiplication des occasions de rencontres, d'échanges et parfois de confrontations entre praticiens et chercheurs dans le domaine de la communication des organisations, invite à recenser les raisons qui incitent les différents acteurs concernés à collaborer. Cette situation donne également l'occasion d'ouvrir des questionnements scientifiques d'ordre épistémologique, théorique et pratique. Ce texte introduit enfin les deux parties qui organisent les six contributions du présent dossier. La première partie explore certaines des fertilisations croisées entre théories et pratiques. La deuxième questionne des apports liés à l'adoption d'épistémologies dite de « convention constructiviste ».

Increasing opportunities for meetings, exchanges, and occasional confrontations between practitioners and researchers in the field of organizational communication invite us to take stock of the reasons that encourage the various actors to collaborate. It also gives us the chance to 
address research issues of an epistemological, theoretical, and practical nature. This paper introduces the two parts in which the six contributions to this special issue are organized. While the first part explores cross-fertilization between theory and practice, the second questions the input provided by the adoption of constructivist epistemologies.

\section{INDEX}

Keywords : epistemology, theory, practice, communication, organization

Mots-clés : épistémologie, théorie, pratique, communication, organisation

\section{AUTEURS}

\section{LAURENT MORILLON}

LERASS (EA 827) - Université Paul Sabatier, Toulouse 3

Laurent Morillon est maître de conférences en sciences de l'information et de la communication à l'Université de Toulouse (France). Membre du LERASS (EA827), équipe organicom, ses recherches portent sur la communication organisationnelle et les pratiques professionnelles. Adresse électronique : laurent.morillon@iut-tlse3.fr.

\section{ARLETTE BOUZON}

LERASS (EA 827) - Université Paul Sabatier, Toulouse 3

Arlette Bouzon est professeur de sciences de l'information et de la communication, enseigne actuellement à l'Université Paul Sabatier, Toulouse 3 (France). Elle est membre du LERASS (Laboratoire d'Études et de Recherche Appliquées en Sciences Sociales) et anime l'équipe Organicom. Ses travaux portent sur les processus communicationnels, la prise de décision dans l'incertain et l'intelligence collective dans les organisations élargies. Adresse électronique : bouzon@lerass.iut-tlse3.fr.

\section{CAROLYNE LEE}

Faculté des Arts, École Culture et Communication - Université de Melbourne

Carolyne Lee est docteur en anglais, senior lecturer et chercheur à l'université de Melbourne, (Australie), dans l'École de Culture et Communication. Ses recherches portent sur le langage des médias, sur la narration et leur rôle en communication. Son dernier et quatrième ouvrage concerne la narration comme les poétiques communicatives. Adresse électronique : carolyne@unimelb.edu.au. 